\title{
Identifying the extent of oral fluid droplets on echocardiographic machine consoles in COVID-19 era
}

\author{
Kenya Kusunose $^{1}\left[\right.$ [D $\cdot$ Kosuke Matsunaga $^{1} \cdot$ Hirotsugu Yamada $^{2} \cdot$ Masataka Sata $^{1}$
}

Received: 24 August 2020 / Revised: 7 September 2020 / Accepted: 10 September 2020 / Published online: 18 September 2020

(c) Japanese Society of Echocardiography 2020

Keywords Oral fluid droplets · COVID-19 · Prevention

\section{Introduction}

COVID-19 is an ongoing pandemic worldwide [1]. One possible mechanism for the spread of this virus is an aerial transmission via droplets generated during speech. In the setting of echocardiography, the risk of infection is particularly high because observers must be in close proximity to the patient. Thus, personal protection is crucial in the COVID-19 era [2]. An important infection prevention method is equipment care. The machine console is where the observer touches the most, but the extent of where droplets could possibly spread is not well examined. The aim of this study was to identify the extent of oral fluid droplet spread on echocardiographic machine consoles, after observers were speaking in English or Japanese.

\section{Methods}

To count the droplets generated during speech on the console, we utilized liquid riboflavin (vitamin $\mathrm{B}_{2}$ ). Adding 1 tablet riboflavin $(14 \mathrm{mg})$ to water $(150 \mathrm{ml})$ and mixing it created a liquid which emitted bright green light when exposed to handheld ultraviolet lamps ( $365 \mathrm{~nm}$ ). Two observers without surgical masks repeated the words "inhale, exhale, hold" (the words often used during examinations) in English and Japanese, in front of echocardiographic machine (EUB7500, Hitachi Medical Corporation, Japan) after ingesting

Kenya Kusunose

kusunosek@tokushima-u.ac.jp

1 Department of Cardiovascular Medicine, Tokushima University Hospital, 2-50-1 Kuramoto, Tokushima, Japan

2 Department of Community Medicine for Cardiology, Tokushima University Graduate School of Biomedical Sciences, Tokushima, Japan the liquid with volunteers lying on your left side echocardiography. The distance between the observer's face and the echocardiographic machine console was $50-60 \mathrm{~cm}$. The surface of the console was divided into 16 equal segments, and droplets were counted in each part. This process was repeated ten times for each observer and the droplets were counted. Continuous data are expressed as the mean \pm standard deviation. Finally, two observers with surgical masks repeated the same words to check the droplets on the surface of the console. We compared the number of droplets between English and Japanese using an unpaired Student's $t$-test. All statistical analyses were performed with SPSS 25.0 (IBM Corp., Armonk, NY, USA) and the figure was made by Origin Software 2020b (Lightstone Corp., Tokyo, Japan). $p<0.05$ was considered statistically significant.

\section{Results}

When surgical masks were not worn, the total counts of droplets were 682 for Japanese and 717 for English, with no significant difference in the counts of droplets for each segment between the two languages (Table 1 all $p>0.05$ ). Figure 1a shows the examples of droplets with fluorescent lamp and ultraviolet lamp. All droplets were clearly detected by the ultraviolet lamp and many droplets (over 20 counts) were observed after three repetitions of the same speech. Figure $1 \mathrm{~b}$ shows the distribution map for the droplets on the console of ultrasound machine. The place where most droplets were counted was at the front right side in both languages. The droplets after English speech reached slightly farther than those after Japanese.

It was also important that no droplets were detected on the console when surgical masks were worn in this setting, although it is unlikely that examinations would be 
Table 1 Averaged number of droplets on the console of the ultrasound machine when surgical masks were not worn

\begin{tabular}{lccc}
\hline & English & Japanese & $p$ value \\
\hline Segment 1 & $4.1 \pm 2.9$ & $3.9 \pm 1.6$ & 0.74 \\
Segment 2 & $7.8 \pm 4.7$ & $7.9 \pm 3.2$ & 0.94 \\
Segment 3 & $6.2 \pm 3.4$ & $5.6 \pm 3.1$ & 0.54 \\
Segment 4 & $2.9 \pm 2.5$ & $3.8 \pm 2.6$ & 0.30 \\
Segment 5 & $1.5 \pm 1.7$ & $1.6 \pm 1.5$ & 0.84 \\
Segment 6 & $3.5 \pm 2.0$ & $4.0 \pm 2.5$ & 0.49 \\
Segment 7 & $2.5 \pm 1.8$ & $2.3 \pm 1.3$ & 0.70 \\
Segment 8 & $1.3 \pm 1.3$ & $1.2 \pm 1.2$ & 0.80 \\
Segment 9 & $0.8 \pm 1.4$ & $0.7 \pm 0.9$ & 0.78 \\
Segment 10 & $1.1 \pm 1.6$ & $1.7 \pm 1.8$ & 0.26 \\
Segment 11 & $1.1 \pm 1.1$ & $1.1 \pm 1.4$ & 0.90 \\
Segment 12 & $0.3 \pm 0.6$ & $0.5 \pm 0.8$ & 0.38 \\
Segment 13 & $0.2 \pm 0.5$ & $0.1 \pm 0.3$ & 0.70 \\
Segment 14 & $0.7 \pm 0.9$ & $0.9 \pm 1.2$ & 0.66 \\
Segment 15 & $0.3 \pm 0.7$ & $0.7 \pm 0.9$ & 0.11 \\
Segment 16 & $0.1 \pm 0.3$ & $0.2 \pm 0.4$ & 0.39 \\
\hline
\end{tabular}

performed with either the sonographer/cardiologist scanning or the patient not wearing a mask in the current COVID era.

\section{Discussion}

The main findings of this study were (1) many droplets were spread by speech, with the front right side of the console particularly contaminated; (2) there was no significant difference for the count of droplets between English and Japanese; (3) mask usage can significantly cut down droplet spreading by speech. To the best of our knowledge, this is the first report to assess the spread of the droplets generated by speech on the surface of an echocardiographic machine using visualized methods. The recent study showed that the "th" sound in the word "healthy" was a significant generator of speech droplets [3]. In our study, the "ex" sound in the word "exhale" and the "d" sound in the word "hold" easily generate droplets. We may need to pay attention to what or how we speak when performing echocardiographic exams.

Our model assessed the potential of dispersing viruses by sonographer or echocardiography with COVID-19 in front of the echo machine. We could not assess the droplets generated during speech by patients infected with COVID19. Although the real object of interest would be droplet reach/distribution emanating from the patient, the actual spread range of the droplets exhaled by the patient remains unclear. We did not assess aerosols because special tools are needed to visually detect micro-sized droplets. Considering the existence of smaller droplets and aerosols, the actual reach of droplets can expect to be much wider in the clinical setting.

The important thing is that all personnel involved in performing the echocardiogram of a patient with COVID-19 or at risk for having COVID-19 wear masks in addition to other personal protective equipment. When they and the patient are wearing masks, there is reduced risk of the passage of the droplets that our experiment generates.

In conclusions, sonographers, physicians, and all other medical staff should strive to decrease their infection risk in the echocardiographic laboratory [4]. Based on our results, it is important to keep the following points in mind when performing echocardiography. (1) The examinations should be performed while wearing a mask. (2) Avoid unnecessary conversation. (3) The contamination area is mainly at the front right side of console. 

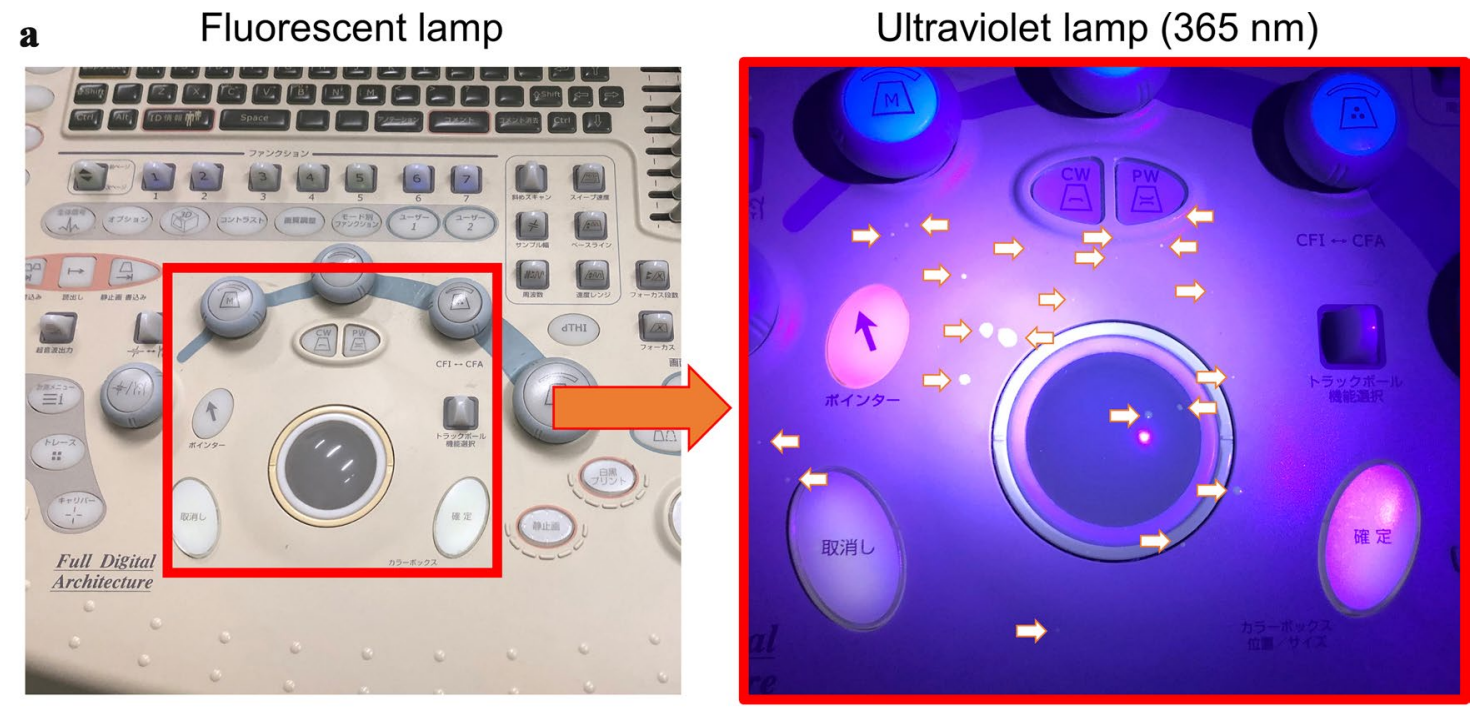

b

Distribution map for droplets in English

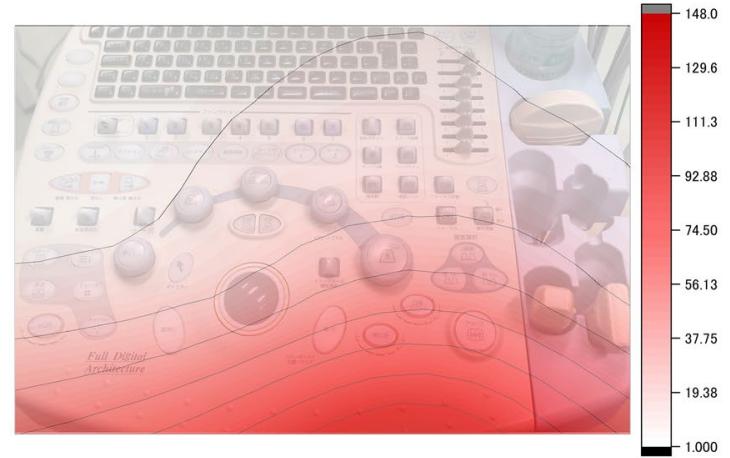

Distribution map for droplets in Japanese

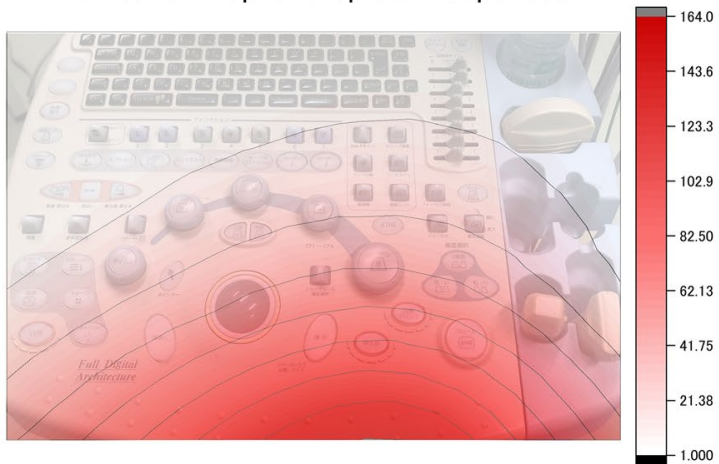

Fig. 1 Examples of droplets (white arrows) with ultraviolet lamp (a) and the distribution map for droplets on the console of the ultrasound machine in English and Japanese (b), when surgical masks were not worn

Author contributions $\mathrm{KK}$ and $\mathrm{KM}$ wrote the manuscript. KK, KM, HY, and MS conceived the idea and proofread the manuscript. All authors have read and approve the submission.

\section{Funding None.}

\section{Compliance with ethical standards}

Conflict of interest Kenya Kusunose, Kosuke Matsunaga, Hirotsugu Yamada, and Masataka Sata declare that they have no conflict of interest.

Human rights statement and informed consent All procedures followed were in accordance with the ethical standards of the responsible committee on human experimentation (institutional and national) and with the Helsinki declaration of 1964 and later revisions. Due to the anonymous nature of the data, the requirement for informed consent was waived by our Institutional Review Board.

\section{References}

1. Sugimoto T, Mizuno A, Kishi T, et al. Coronavirus disease 2019 (COVID-19) information for cardiologists - systematic literature review and additional analysis. Circ J. 2020;84:1039-43.

2. Kirkpatrick JN, Mitchell C, Taub C, et al. ASE statement on protection of patients and echocardiography service providers during the 2019 novel coronavirus outbreak: endorsed by the American College of Cardiology. J Am Soc Echocardiogr. 2020;33:648-53.

3. Anfinrud P, Stadnytskyi V, Bax CE, et al. Visualizing speechgenerated oral fluid droplets with laser light scattering. N Engl J Med. 2020;382:2061-3.

4. Seo Y, Daimon M, Yamada H, et al. Review of the efforts of the Japanese Society of Echocardiography for coronavirus disease 2019 (COVID-19) during the initial outbreak in Japan. J Echocardiogr. 2020. https://doi.org/10.1007/s12574-020-00487-5.

Publisher's Note Springer Nature remains neutral with regard to jurisdictional claims in published maps and institutional affiliations. 